\title{
Crystal Growth of Isotactic Poly(butene-1) in the Melt. I. Kinetic Roughening
}

\author{
Motoi YAmashita ${ }^{\dagger}$ Hideki MiYAJI, Akitaka Hoshino, and Kunihide IZUMI \\ Department of Physics, Graduate School of Science, Kyoto University, Kyoto 606-8502, Japan
}

(Received October 14, 2003; Accepted December 16, 2003)

\begin{abstract}
The morphology, lateral growth rate and long spacings of isotactic poly(butene-1) (it-PB1) have been investigated for crystallization from the melt over a wide range of crystallization temperature from 50 to $111.9^{\circ} \mathrm{C}$. The morphology of $i t$-PB 1 crystals is rounded shape at crystallization temperatures lower than $85^{\circ} \mathrm{C}$, while lamellar single crystals possess faceted morphology at higher crystallization temperatures; the kinetic roughening transition occurs around $85^{\circ} \mathrm{C}$. The nucleation and growth mechanism for crystallization does not work below $85^{\circ} \mathrm{C}$, since the growth face is rough. However, the growth rate and the long spacings show the supercooling dependence derived from the nucleation and growth mechanism; the nucleation theory seems still to work even for rough surface growth. Taking account of a pinning model revised recently by Toda, possible mechanisms for the crystal growth of polymers are discussed.

KEY WORDS Isotactic Poly(butene-1) / Growth Rate / Morphology / Long Spacings / Kinetic Roughening / Pinning Model / Entropic Barrier /
\end{abstract}

Crystallization of polymers has been investigated through morphology, growth rate and lamellar thickness. The growth rate observed at a crystallization temperature, $T$, is known to be well described by the following equation

$$
G=G_{0} \exp \left[-\frac{U}{R\left(T-T_{\mathrm{V}}\right)}\right] \exp \left[-\frac{K}{T \Delta T}\right]
$$

where $G_{0}$ and $K$ are constants, $U$ is the 'activation' energy for polymer diffusion, $R=k N_{\mathrm{A}}$, ( $k$ is the Boltzmann constant and $N_{\mathrm{A}}$ is Avogadro's number) $T_{\mathrm{V}}$ is the Vogel temperature $\left(=T_{\mathrm{g}}-30(\mathrm{~K}), T_{\mathrm{g}}\right.$ is the glass transition temperature), $\Delta T\left(=T_{\mathrm{m}}^{0}-T\right)$ is a supercooling ( $T_{\mathrm{m}}^{0}$ is the equilibrium melting temperature). The first exponential factor is the VogelFulcher factor for viscosity and the second exponential factor is the surface kinetic factor.

According to the nucleation theory ${ }^{1}$ by Hoffman et $a l$., the secondary two-dimensional nucleation rate $i$ is given by the following equation:

$$
i \propto \exp \left[-\frac{4 b \sigma \sigma_{\mathrm{e}} T_{\mathrm{m}}^{0}}{k \Delta h_{\mathrm{f}} T \Delta T}\right]
$$

where $b$ is the layer thickness, $\sigma$ and $\sigma_{\mathrm{e}}$ the side- and end-surface free energies per unit area, respectively, and $\Delta h_{\mathrm{f}}$ the heat of fusion per unit volume of crystal.

In the regime of multiple nucleation, regime II, the surface kinetic factor of the growth rate is given by the following equation: ${ }^{2}$

$$
G_{\mathrm{II}}=b(2 i g)^{1 / 2}
$$

where $g$ is the propagation velocity of steps on a growth face. Since $g$ is assumed to be almost constant ${ }^{1}$ in the nucleation theory, $K$ in Eq 1 is represented by the following equation

$$
K=\frac{2 b \sigma \sigma_{\mathrm{e}} T_{\mathrm{m}}^{0}}{k \Delta h_{\mathrm{f}}} \equiv K_{\mathrm{II}}
$$

The nucleation theory therefore successfully explains the observed supercooling dependence of growth rate expressed by Eq 1 .

The nucleation theory also gives the observed dependence ${ }^{1}$ of lamellar thickness $l$ on supercooling $\Delta T$ :

$$
l=\frac{A}{\Delta T}+\delta l,
$$

where $A$ and $\delta l$ are constants. According to the theory, $A$ is expressed as follows:

$$
A=\frac{2 \sigma_{\mathrm{e}} T_{\mathrm{m}}^{0}}{\Delta h_{\mathrm{f}}}
$$

The characteristic length, $d$, in the nucleation theory described by $i$ and $g$, is the mean distance between adjacent steps on the growth face and is called the kinetic length: ${ }^{3}$

$$
d=\left(\frac{2 g}{i}\right)^{1 / 2}
$$

As $\Delta T$ increases, $d$ decreases rapidly since $i$ depends on $1 / T \Delta T$ exponentially and $g$ remains almost constant. ${ }^{1}$ When the kinetic length $d$ becomes as small as the order of widths of several stems, the growth face becomes rough and the morphology of lamellar crystals changes from faceted to rounded ones. This

${ }^{\dagger}$ To whom correspondence should be addressed (Tel \& Fax: +81-75-753-3754, E-mail: motoi@ scphys.kyoto-u.ac.jp). 
Table I. Physical property of it-PB1

\begin{tabular}{|c|c|c|c|c|c|c|c|c|c|c|c|}
\hline & \multirow[b]{2}{*}{$\begin{array}{l}T_{\mathrm{g}}{ }^{\mathrm{c}} \\
\left({ }^{\circ} \mathrm{C}\right)\end{array}$} & \multirow[b]{2}{*}{$\begin{array}{c}U^{\mathrm{d}} \\
\left(\mathrm{cal} \mathrm{\textrm {mol } ^ { - 1 }}\right)\end{array}$} & \multirow[b]{2}{*}{$\begin{array}{l}\text { Space } \\
\text { group }\end{array}$} & \multicolumn{3}{|c|}{ Unit cell parameters } & \multirow[b]{2}{*}{$\begin{array}{l}\text { Density } \\
\left(\mathrm{g} \mathrm{cm}^{-3}\right)\end{array}$} & \multicolumn{2}{|c|}{ Stem parameters } & \multirow[b]{2}{*}{$\begin{array}{c}\Delta h_{\mathrm{f}}^{\mathrm{f}} \\
\left(\mathrm{J} \mathrm{m}^{-3}\right)\end{array}$} & \multirow{2}{*}{$\begin{array}{c}\text { Chain } \\
\text { conformation } \\
\text { (monomers/turn) }\end{array}$} \\
\hline & & & & $\begin{array}{l}a_{0} \\
(\AA)\end{array}$ & $\begin{array}{l}b_{0} \\
(\AA)\end{array}$ & $\begin{array}{l}c_{0} \\
(\AA)\end{array}$ & & $\begin{array}{c}a \\
(\AA)\end{array}$ & $\begin{array}{c}b \\
(\AA)\end{array}$ & & \\
\hline Form $\mathrm{I}^{\mathrm{a}}$ & -54.2 & 1500 & $\mathrm{R} \overline{3} \mathrm{c}$ & 17.7 & 17.7 & 6.5 & 0.95 & & & $1.35 \times 10^{8}$ & $3 / 1$ \\
\hline Form II $^{\mathrm{b}}$ & & & $\mathrm{P} \overline{4} \mathrm{~b} 2$ & 14.6 & 14.6 & 21.2 & 0.888 & 7.3 & 7.3 & $1.09 \times 10^{8}$ & $11 / 3$ \\
\hline
\end{tabular}

$1 \AA=10^{-10} \mathrm{~m}$. ${ }^{\mathrm{a}}$ From Ref $11{ }^{\mathrm{b}}$ From Ref $12{ }^{\mathrm{c}}$ From Ref $13{ }^{\mathrm{d}}$ From Ref $14{ }^{\mathrm{e}}$ From Ref $15{ }^{\mathrm{f}}$ From Ref 16

morphological change is called kinetic roughening transition. Nucleation theory assumes flat growth faces, i.e., facets, as its prerequisite, on which nucleation and growth occur. However, the rough surfaces of crystals with rounded morphology do not satisfy this essential prerequisite condition for the nucleation theory; instead, adhesive growth generally takes place on rough growth faces. ${ }^{4}$ Hence, the kinetic roughening transition should accompany a deviation from nucleation controlled growth: the growth mechanism changes from nucleation controlled growth to adhesive growth.

However, a paradox has been reported on the crystallization of isotactic polystyrene (it-PS) in solutions and in the melt by Tanzawa et al. ${ }^{5,6}$ While the morphology changes from the faceted to rounded morphology through the kinetic roughening temperature with decreasing temperature, the growth rate does not show any transition at the kinetic roughening temperature.

In order to address this paradox, we have focused attention on the crystallization of isotactic poly(butene-1) (it-PB1) in the melt. It-PB1 has two polymorphs, ${ }^{7-10}$ the trigonal form (I) and the tetragonal form (II), as shown in Table I. The crystal of form II grows much faster than that of form I in the melt. However, form I is stable and form II is metastable. Accordingly, the transformation to form I then takes place in several days after cooling to room temperature. By comparing the growth rates of the two different forms in the melt, one could elucidate the kinetic factor intrinsic to each phase in Eq 1 to determine the validity of a new theory capable of explaining the mechanisms that give rise to the paradoxical relationship between the growth rate and the morphology.

We have investigated the crystallization of it-PB1 form II crystals in the melt. Also, we have succeeded in measuring the growth rate and morphology of itPB1 form I single crystals grown in the melt. In this paper, we focus on the crystallization of the form II crystals, and report the growth rate, lamellar thickness and morphology of $i t$-PB1 form II crystals grown in the melt. The paradox between $\Delta T$ dependence of growth rate and the morphology is confirmed on the crystallization of $i t$-PB1. We will discuss the paradox on the basis of a pinning model revived recently by Toda. ${ }^{17}$

\section{EXPERIMENTAL}

The it-PB1s used in this study were purchased either from Scientific Polymer Product (sample A: $\bar{M}_{\text {w }}=185000$; the melt index is $\left.20 \mathrm{~g} / 10 \mathrm{~min}\right)$, or Shell (sample B: the melt index is $20 \mathrm{~g} / 10 \mathrm{~min}$ ).

\section{Growth Rate}

In-situ observations of the crystallization process were carried out using an optical microscope (Nikon Corp. OPTIPHOT2) with a hot-stage (Mettler Co., Ltd., FP82). Films of $i t$-PB1, ca. $50 \mu \mathrm{m}$ thick, between two cover glasses were melted at $140^{\circ} \mathrm{C}$ for $2 \mathrm{~min}$ and cooled to a crystallization temperature between $52^{\circ} \mathrm{C}$ and $111.9^{\circ} \mathrm{C}$. The growth rate was determined from the time dependence of the radius of spherulites or the major axis of axialites.

\section{Morphology}

Thin it-PB1 films were prepared by casting a $p$ xylene solution $(0.1 \mathrm{wt} \%$ it-PB1) onto a carbon-coated mica. The films dried was heated up to $140^{\circ} \mathrm{C}$, cooled to a crystallization temperature and crystallized in the hot-stage for a suitable time, and quenched to room temperature. The it-PB1-carbon films were floated on a water surface and picked up on electron microscope grids. The it-PB1 crystals on the carbon film were observed by transmission electron microscopy (TEM; JEOL Ltd., JEM-1200EX II) and optical microscopy (OM) to investigate the morphology of crystals.

\section{Long Spacings}

Small angle X-Ray scattering (SAXS) photographs were taken with a SAXS camera (camera length $414 \mathrm{~mm}$ ) in vacuum to obtain lamellar long spacings, using an imaging plate system (Rigaku K.K., R-AXIS DSII). X-Ray used was nickel-filtered $\mathrm{CuK} \alpha$ radiation generated at $50 \mathrm{kV}$ and $140 \mathrm{~mA}$. After the subtraction of the background intensity, isotropic two-dimensional data were circularly averaged to obtain one-dimensional data, and corrected for the Lorentz factor. Films 
of $i t$-PB1, about $500 \mu \mathrm{m}$ in thickness, between aluminum foil were melted at $150^{\circ} \mathrm{C}$ for $3 \mathrm{~min}$ in an oven, transferred quickly to the hot-stage kept at a crystallization temperature. The films crystallized were left at room temperature for $10 \mathrm{~d}$ and used for SAXS measurements. In $10 \mathrm{~d}$ at room temperature the spontaneous form II to I phase transition occurs without changing the lamellar thickness of the crystals. Since the crystal density of form I is larger than that of form II, SAXS intensity is much enhanced after $10 \mathrm{~d}$.

To obtain long spacings just before the melting, we carried out annealing for samples crystallized at 40, $70,80,90$, and $100^{\circ} \mathrm{C}$, and observed thermal thickening of lamellar crystals; samples were crystallized in the same condition as that of samples without annealing, transferred to another hot stage (Linkam Co., Ltd., LK-FDCS II), heated to the melting points determined by DSC measurements in Melting Point Temperature at a rate of $30 \mathrm{~K} \mathrm{~min}^{-1}$, and cooled to room temperature at a rate of $50 \mathrm{~K} \mathrm{~min}^{-1}$. The annealed samples left at room temperature for $10 \mathrm{~d}$ were used for SAXS measurements.

\section{Melting Point Temperature}

Differential scanning calorimetry was performed (Rigaku K.K., DSC-10A) at a heating rate of $30 \mathrm{~K} \mathrm{~min}^{-1}$. The melting temperature of form II was determined from the onset temperature of the endothermic peak. Samples used for DSC were crystallized under the same condition as that of samples for SAXS, and preserved in a freezer to prevent the phase transition.

\section{Identification of Crystal Structure}

Wide angle X-Ray scattering (WAXS) was per- formed to identify crystal structures, using an imaging plate system (Rigaku K.K., R-AXIS DSII). Isotropic two-dimensional intensity collected was averaged to give the one-dimensional data, and corrected for the Lorentz factor. X-Ray used was nickel-filtered $\mathrm{Cu}$ $\mathrm{K} \alpha$ radiation. Samples were crystallized under the same condition as that of samples for SAXS. They were used for WAXS measurements both immediately after crystallization and after $10 \mathrm{~d}$ of storage at room temperature.

\section{RESULTS}

\section{Crystal Structure from WAXS}

We confirmed by WAXS measurements that samples immediately after the crystallization are in the form II. Hence, the observed growth rate, long spacings and crystal morphology are those of crystals in form II. Samples stored at room temperature for $10 \mathrm{~d}$ after crystallization exhibited peaks characteristic of form I.

\section{Melting Temperature}

Table II lists the DSC and SAXS results for sample A: crystallization and melting temperatures, the values of the first- and second-order long spacings, and the ratios of the first-order long spacings to the second-order long spacings for samples before and after the annealing. The differences between the values of the first-order long spacings of samples before and after annealing are also listed to illustrate thermal thickening effect. Both of the first- and second-order reflections were observed in the wide range of crystallization temperature from 50 to $90^{\circ} \mathrm{C}$. The ratios of the first-order long spacings to the second-order

Table II. DSC and SAXS ${ }^{\mathrm{a}}$ results

\begin{tabular}{|c|c|c|c|c|c|c|c|c|}
\hline \multirow[b]{2}{*}{$\begin{array}{c}T_{\mathrm{c}} \\
\left({ }^{\circ} \mathrm{C}\right)\end{array}$} & \multirow[b]{2}{*}{$\begin{array}{c}T_{\mathrm{m}} \\
\left({ }^{\circ} \mathrm{C}\right)\end{array}$} & \multicolumn{3}{|c|}{ After annealing } & \multicolumn{3}{|c|}{ Before annealing } & \multirow[b]{2}{*}{$\begin{array}{c}\Delta L \\
(\AA)\end{array}$} \\
\hline & & $\begin{array}{l}L_{1} \\
(\AA)\end{array}$ & $\begin{array}{c}L_{2} \\
(\AA)\end{array}$ & $\begin{array}{c}L_{1} / L_{2} \\
(-)\end{array}$ & $\begin{array}{l}L_{1} \\
(\AA)\end{array}$ & $\begin{array}{c}L_{2} \\
(\AA)\end{array}$ & $\begin{array}{c}L_{1} / L_{2} \\
(-)\end{array}$ & \\
\hline 40.3 & 102.8 & 313 & 155 & 2.02 & 227 & - & - & 86 \\
\hline 50 & & & & & 241 & 130 & 1.85 & \\
\hline 60 & & & & & 263 & 139 & 1.90 & \\
\hline 65 & & & & & 263 & 147 & 1.79 & \\
\hline 70 & 104.1 & 327 & 164 & 2 & 283 & 155 & 1.83 & 44 \\
\hline 75 & & & & & 307 & 158 & 1.94 & \\
\hline 80 & 104.6 & 342 & 169 & 2.02 & 313 & 164 & 1.91 & 29 \\
\hline 85 & & & & & 342 & 171 & 2.00 & \\
\hline 90 & 106.5 & 378 & 186 & 2.03 & 378 & 186 & 2.03 & 0 \\
\hline 95 & & & & & $(398)^{\mathrm{b}}$ & 196 & - & \\
\hline 100 & 111.2 & $(452)^{\mathrm{b}}$ & 223 & 一 & $(459)^{\mathrm{b}}$ & 227 & - & 一 \\
\hline
\end{tabular}

${ }^{a}$ The first-order long spacings, $L_{1}$, the second-order long spacings, $L_{2}$ the ratio of the first-order to the second order $L_{1} / L_{2}$ are given for both before and after annealing. Also given are the difference $\Delta L_{1}$ between the values of the first-order long spacings of samples before and after annealing. ${ }^{b}$ Values in brackets were calculated first-order long spacings from the second-order long spacings. 
long spacings are about two. For samples crystallized at $90^{\circ} \mathrm{C}$, this ratio is 2.03 . At 95 and $100^{\circ} \mathrm{C}$, only the second-order reflections were capable of being observed. By multiplying the second-order long spacings by the ratio at $90^{\circ} \mathrm{C}, 2.03$, we calculated the first-order long spacings at 95 and $100^{\circ} \mathrm{C}$.

At 40,70 , and $80^{\circ} \mathrm{C}$, the first-order long spacings of annealed samples have larger values than those for samples before thermal treatment. The difference becomes larger as the crystallization temperature is decreased. This difference is due to the effect of thickening in annealing; the lamellae in samples crystallized at lower temperatures thickens at the annealing, giving the larger values of long spacings. Samples crystallized at lower temperatures have thinner lamellae. The sliding diffusion of chain segments along the chain axis occurs more easily for thinner lamellae than for thicker lamellae, to give rise to the thickening of lamellae. For samples crystallized at 90 and $100^{\circ} \mathrm{C}$, lamellae are too thick for this sliding motion to occur, resulting in the same long spacing values for samples before and after the annealing.

The ratio of the first-order to the second-order long spacing for the annealed sample has a value closer to 2.0 than that of the samples before annealing. This is due to the narrower distribution of long spacings caused by the annealing.

The melting temperature $T_{\mathrm{m}}(l)$ of crystals with lamellar thickness $l$ is expressed by the Gibbs-Thomson equation:

$$
T_{\mathrm{m}}(l)=T_{\mathrm{m}}^{0}\left[1-\frac{2 \sigma_{\mathrm{e}}}{\Delta h_{\mathrm{f}} l}\right]
$$

Figure 1 shows the inverse of long spacing dependence of melting temperature for samples before and after annealing. The data for samples before annealing evidently deviates from the linearity. The deviation is

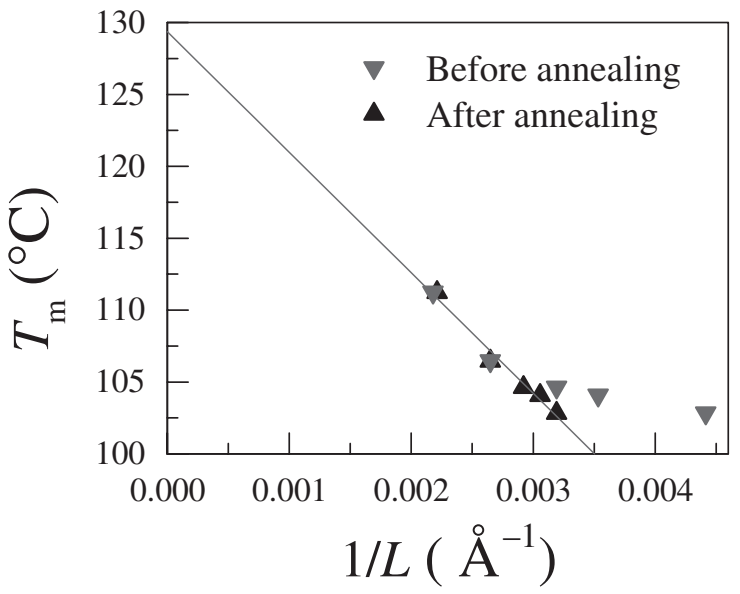

Figure 1. Dependence of the melting temperature $T_{\mathrm{m}}$ on the reciprocal long spacings $1 / L$ for samples $(\boldsymbol{\nabla})$ before and $(\boldsymbol{\Delta})$ after annealing. attributed to the thickening during the DSC measurements; lamellae in samples crystallized at 40, 70, $80^{\circ} \mathrm{C}$ thickens during heating up to their melting temperatures.

The data for annealed samples plausibly obey the linear relation given in $\mathrm{Eq} 8$ to give the equilibrium melting point temperature $T_{\mathrm{m}}^{0}=129.4^{\circ} \mathrm{C}$ for an infinite thick crystal. The slope gives the value of $2 \sigma_{\mathrm{e}} T_{\mathrm{m}}^{0} / \Delta h_{\mathrm{f}}=8.38 \times 10^{3} \AA \mathrm{K}\left(1 \AA=10^{-10} \mathrm{~m}\right)$, assuming the degree of crystallity is 1.0 . The value of $\sigma_{\mathrm{e}} / \Delta h_{\mathrm{f}}$ is obtained to be $1.04 \times 10^{-9} \mathrm{~m} ; \sigma_{\mathrm{e}}$ is estimated as $1.14 \times 10^{-1} \mathrm{~J} \mathrm{~m}^{-2}$, and chain folding free energy $q=2 a b \sigma_{\mathrm{e}}$ as $1.21 \times 10^{-19} \mathrm{~J} / \mathrm{stem}$ by use of the parameters in Table I.

\section{Long Spacings}

Figure 2 shows the first-order long spacings before annealing as the function of the inverse supercooling, $1 / \Delta T$, for sample A. Here, $\Delta T=T_{\mathrm{m}}^{0}-T$. The $1 / \Delta T$ dependence of long spacings demonstrates linearity over the whole temperature range investigated; Eq 5 holds from 40 to $100^{\circ} \mathrm{C}$.

According to the Eqs 5 and 6, the extrapolation to $1 / \Delta T=0 \mathrm{~K}^{-1}$ of the straight line in Figure 2 gives $\delta l=114 \AA$, and from the slope the value of $2 \sigma_{\mathrm{e}} T_{\mathrm{m}}^{0} / \Delta h_{\mathrm{f}}$ is obtained to be $1.01 \times 10^{4} \AA \mathrm{K}$, assuming the degree of crystallinity is $1.0 ; \sigma_{\mathrm{e}}$ is estimated as $1.36 \times 10^{-1} \mathrm{~J} \mathrm{~m}^{-2}$, using the value of $\Delta h_{\mathrm{f}}$ given in Table I and the equilibrium melting temperature $T_{\mathrm{m}}^{0}$ we determined.

It is to be noted that $\sigma_{\mathrm{e}}$ obtained here on the basis of the nucleation theory is in good agreement with that obtained from melting temperature versus reciprocal long spacings plot; the nucleation theory seems to work well for crystallization temperature dependence of the lamellar thickness.

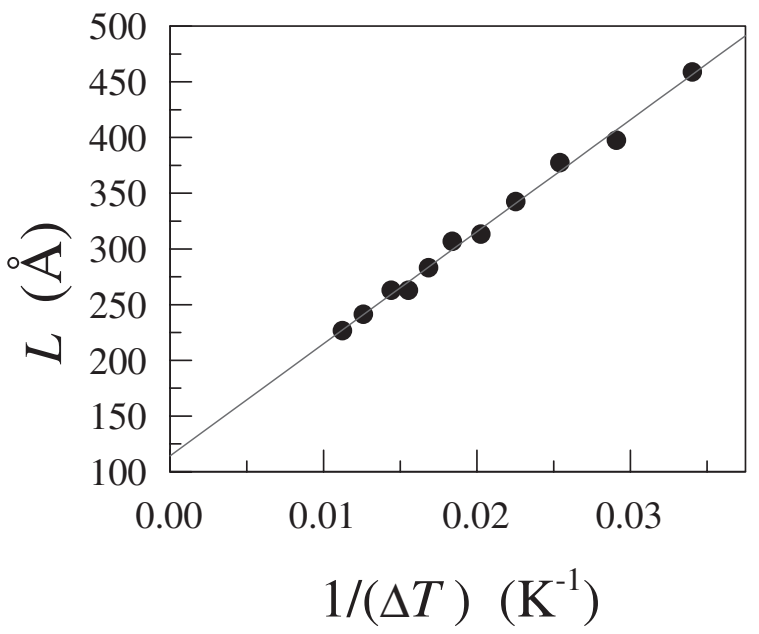

Figure 2. Long spacings $L$ plotted against the reciprocal supercooling $1 / \Delta T$. 
(a)

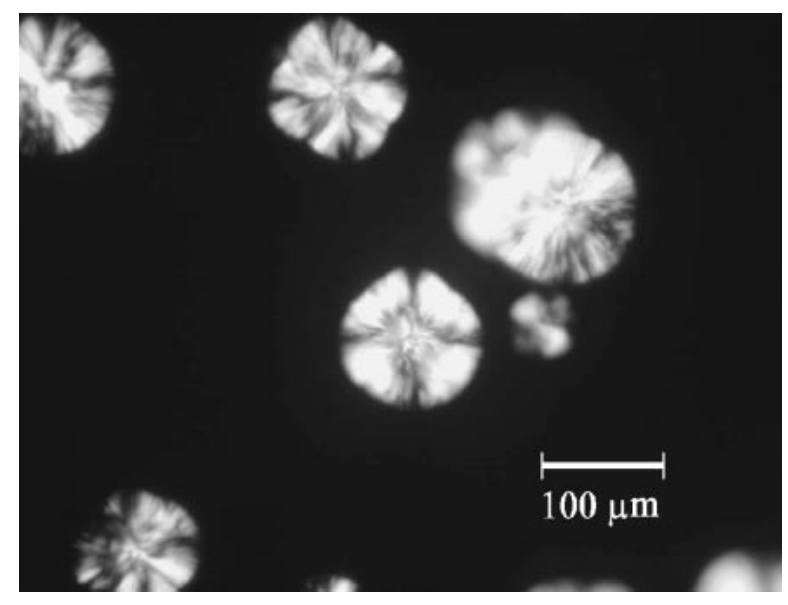

(b)

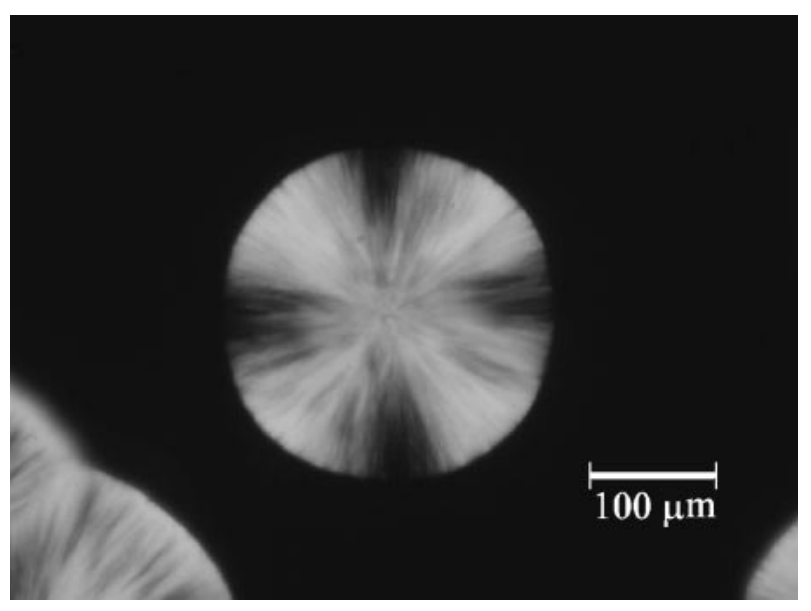

Figure 3. Optical micrographs of it-PB1 crystallized in the melt at $90{ }^{\circ} \mathrm{C}$; crossed polars. (a) $4 \mathrm{~min}$ and (b) $10 \mathrm{~min}$ after the temperature reached $90^{\circ} \mathrm{C}$.

\section{Growth Rate}

Typical optical micrographs of it-PB1 crystals grown from the melt are shown in Figure 3 for sample A. At crystallization temperatures higher than $90^{\circ} \mathrm{C}$, tetragonal, octagonal, oval and circular crystals were usually observed; they are axialites, some of which grew to be circular crystals. At low crystallization temperatures, spherulites were always observed. The size of the crystals $2 R$, i.e., the diameter of spherulites or the major axis of axialites, was measured as a function of time $t$.

The radius of the spherulites or the axialites $R$ increased linearly with crystallization time $t$ for all crystallization temperatures as shown in Figure 4. The growth rate $G$ was determined from the slope of the time-radius curve. The logarithm of $G$ is plotted against crystallization temperature in Figure 5 for samples $\mathrm{A}$ and $\mathrm{B}$. The values of $G$ observed for samples A and B agrees well. The results by Icenogle ${ }^{18}$ are included for the sake of comparison. The $\log G-$ $T$ curve is a half of the typical dome shape.
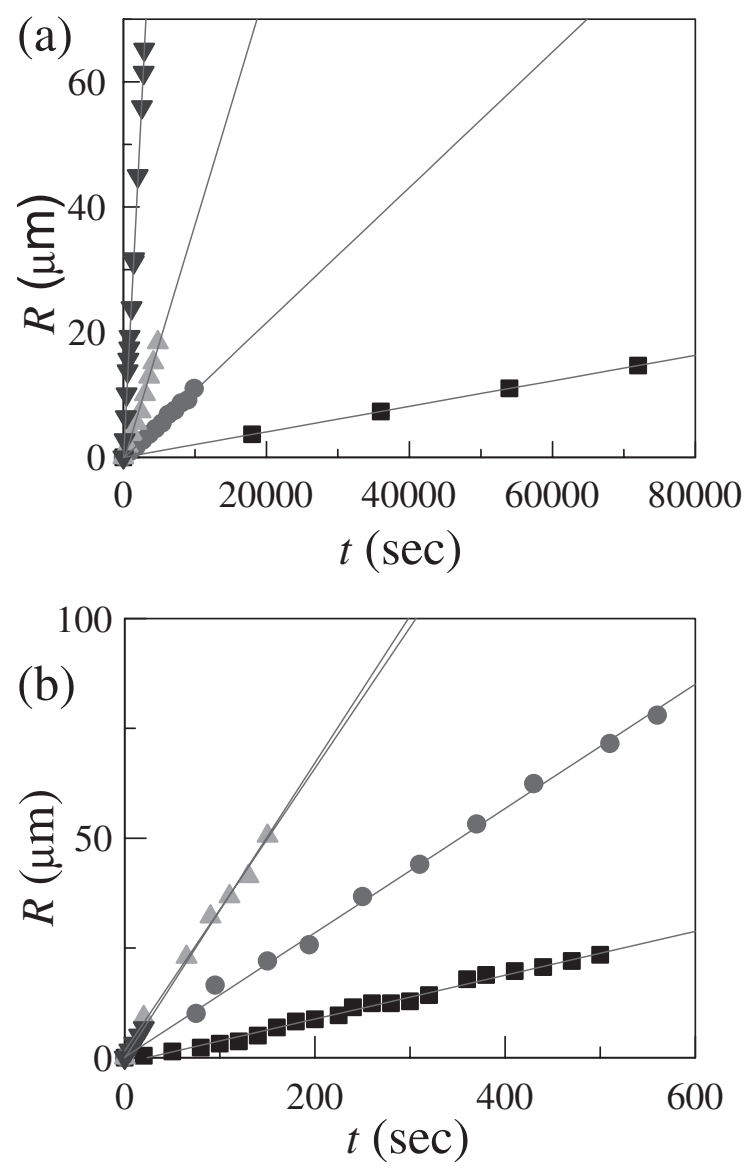

Figure 4. Time dependence of radius $R$ for several crystallization temperatures for sample B. (a): (ם) $111.9^{\circ} \mathrm{C}$, () $108^{\circ} \mathrm{C}$,

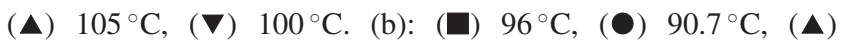
$82.7^{\circ} \mathrm{C},(\boldsymbol{\nabla}) 60^{\circ} \mathrm{C}$.

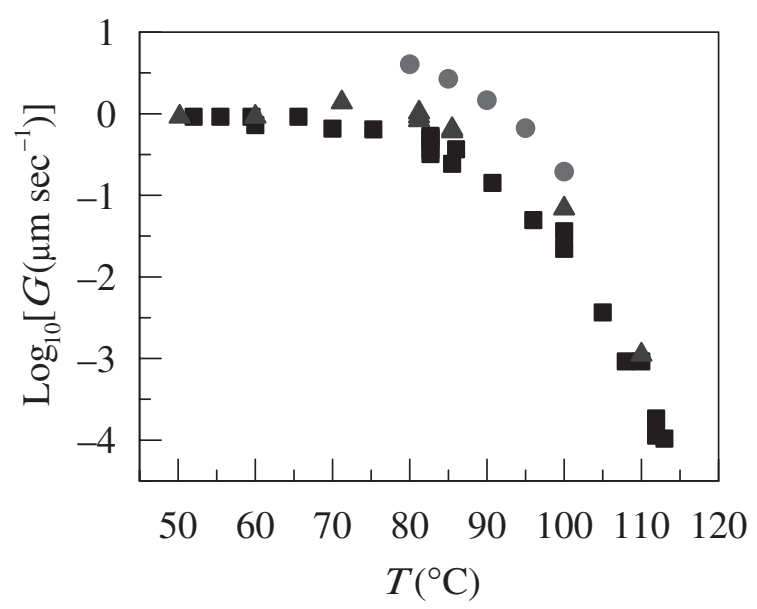

Figure 5. Growth rate $G$ versus crystallization temperature $T$ :

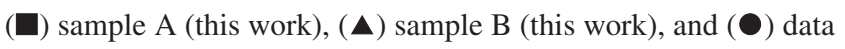
by Icenogle (Ref 11).

Figure 6 shows $\ln G+U / R\left(T-T_{\mathrm{V}}\right)$ as a function of $1 / T \Delta T \cdot \ln G+U / R\left(T-T_{\mathrm{V}}\right)$ depends on $1 / T \Delta T$ linearly over the whole range examined; Eq 1 holds for all the crystallization temperature range investigated from 52 to $111.9^{\circ} \mathrm{C}$.

According to Eq 1, the extrapolation to zero of the 


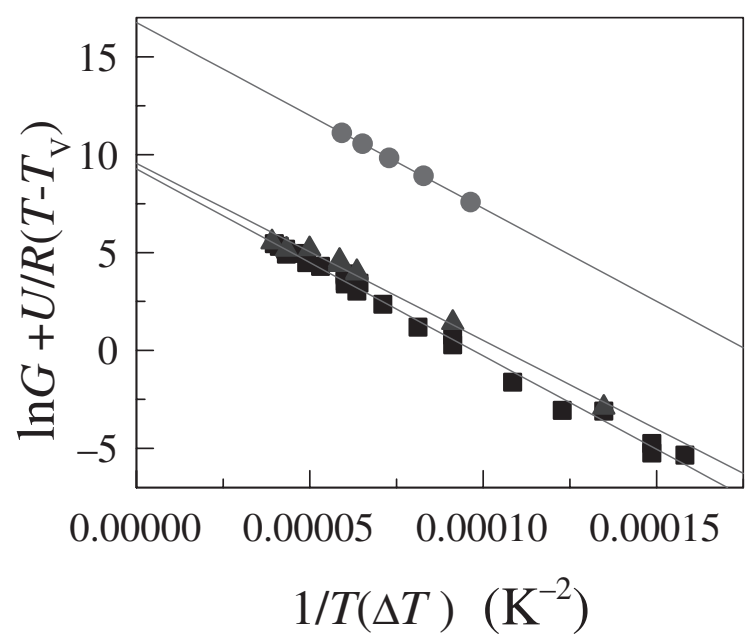

Figure 6. Plot of $\ln G+U / R\left(T-T_{\mathrm{V}}\right)$ vs. $1 / T \Delta T$. Symbols are the same as in Figure 5.

straight line in Figure 6 for $1 / T \Delta T$ gives the values of $G_{0}=1.41 \times 10^{4} \mu \mathrm{m} \mathrm{s}^{-1}$ for sample A and $G_{0}=$ $1.05 \times 10^{4} \mu \mathrm{m} \mathrm{s}^{-1}$ for sample B; from the slope the values of $K$ is obtained to be $9.04 \times 10^{4} \mathrm{~K}^{2}$ for sample $\mathrm{A}$ and $9.54 \times 10^{4} \mathrm{~K}^{2}$ for sample B. Both the values of $G_{0}$ and $K$ agree well for sample A and B.

The values of $G_{0}$ and $K$ obtained from the data by Icenogle are $1.86 \times 10^{4} \mu \mathrm{m} \mathrm{s}^{-1}$ and $9.49 \times 10^{4} \mathrm{~K}^{2}$, respectively. The value of $K$ agrees well with the present result. The difference in the values of $G_{0}$ can be attributed to that of the molecular weight $\left(\bar{M}_{\mathrm{w}}=\right.$ 750000).

From the value of $K$ obtained for sample B and the parameters in Table $\mathrm{I}$, the value of $\sigma \sigma_{\mathrm{e}}$ is calculated as $2.45 \times 10^{-4} \mathrm{~J}^{2} \mathrm{~m}^{-4}$, for regime II; using the value of $\sigma_{\mathrm{e}}$ obtained from the $T_{\mathrm{m}}$ versus $1 / L$ plot, the value of $\sigma$ is estimated as $2.15 \times 10^{-3} \mathrm{~J} \mathrm{~m}^{-2}$.

\section{Morphology}

Figure 7 shows the electron micrograph and the electron diffraction pattern of a single crystal grown at $110^{\circ} \mathrm{C}$. Schematic illustration of the lateral habit of the single crystal is also shown. The electron diffraction pattern from the form II (tetragonal phase) shows the single-crystal net pattern (four 200 reflections and four 220 reflections). The electron diffraction pattern from the form I (trigonal phase) shows arcs (110 reflections (innermost), 300 reflections (middle) and 220 reflections (outermost)). The arcs are attributed to the orientational distribution of small domains of form I crystals transformed from the form II single crystal. ${ }^{19}$ The presence of twelve 110 diffraction arcs, rather than six in Figure 7 is due to the presence of the two orientations of transformation.

There appears a square-shaped crystal with serrated edges in the electron micrograph (Figure 7c). The electron diffraction pattern reveals the crystal is a (a)

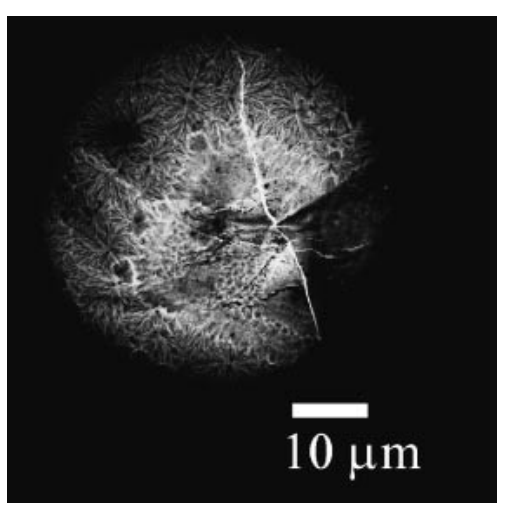

(b)

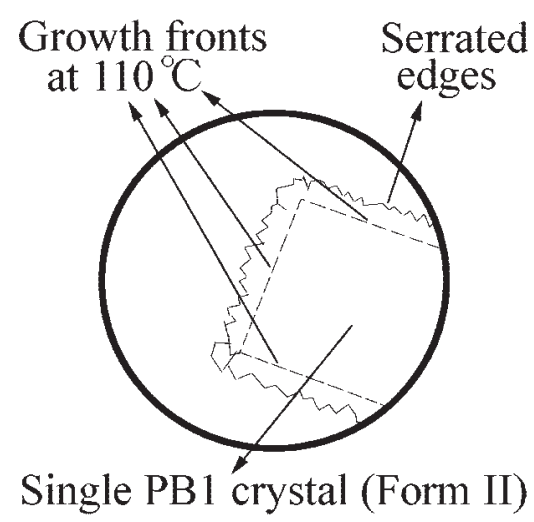

(c)

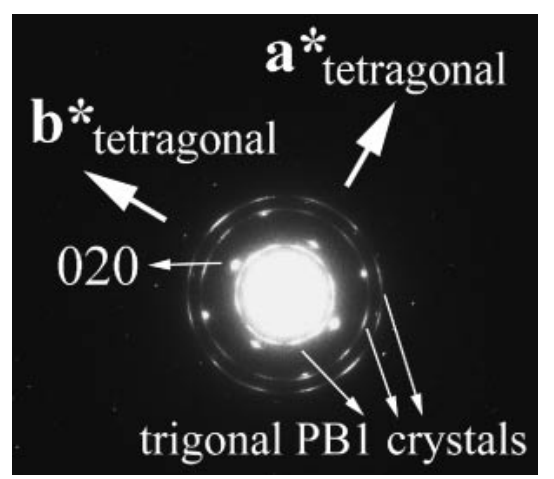

Figure 7. (a) Electron micrograph, (b) schematic illustration, (c) diffraction pattern of an it-PB1 single crystal grown at $110^{\circ} \mathrm{C}$. Thin straight boundary lines observed inside the serrated edges, which are represented by broken lines in the schematic illustration, show the change in lamellar thickness caused by quenching. The region between the boundary lines and the serrated edges is grown during the quenching treatment, and thereby is thinner than that inside the boundary. The boundary lines correspond to the growth faces of the single crystal just before quenching, and these lines show the well faceted growth faces at $110^{\circ} \mathrm{C}$. The region out of the edges is filled with small spherulites formed through quenching; the serrated out line is caused by the instability of growth face at higher supercooling during quenching.

flat-on form II (tetragonal) single crystal. Thin straight boundary lines observed inside the serrated edges show the change in lamellar thickness caused by quenching (they are represented by broken lines in 
the schematic illustration), and thereby correspond to the growth fronts of the single crystal just before quenching. The trace of growth front shows that the form II single crystal is well faceted at $110^{\circ} \mathrm{C}$. We confirmed by electron diffraction that the boundary lines are $\{100\}$ planes. The serrated outline is caused by the instability of growth face at high supercooling during quenching. These facts indicate that the form II single crystal is well facetted at $110^{\circ} \mathrm{C}$.

Figure 8 shows the electron micrograph and diffrac-

(a)

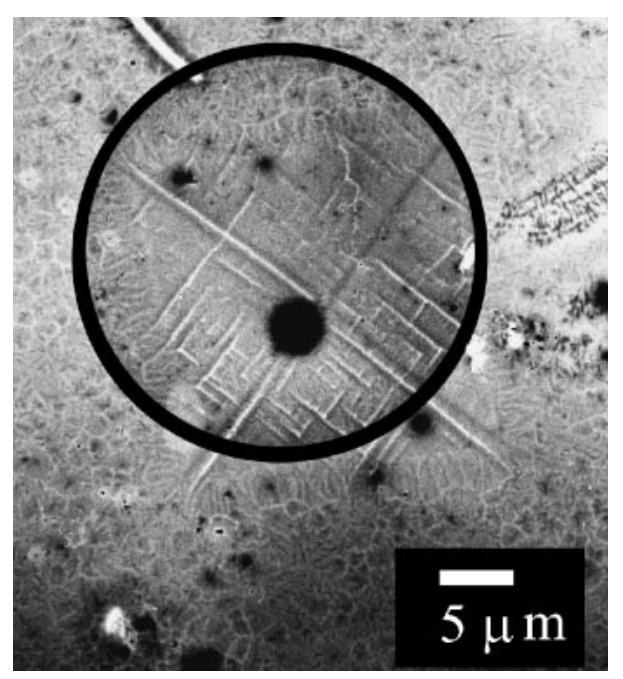

(b)

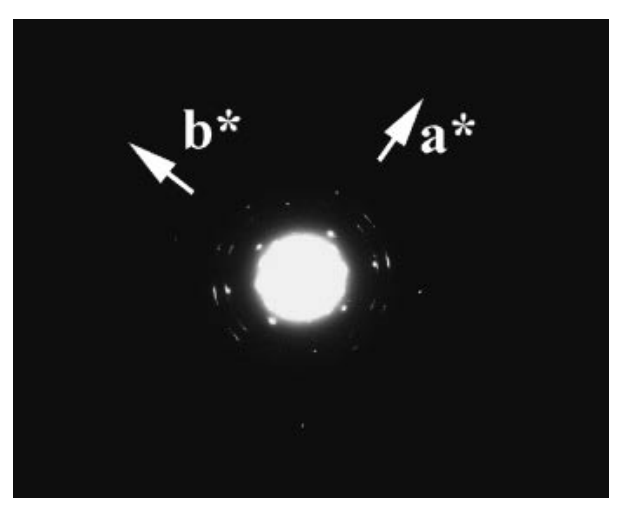

(c)

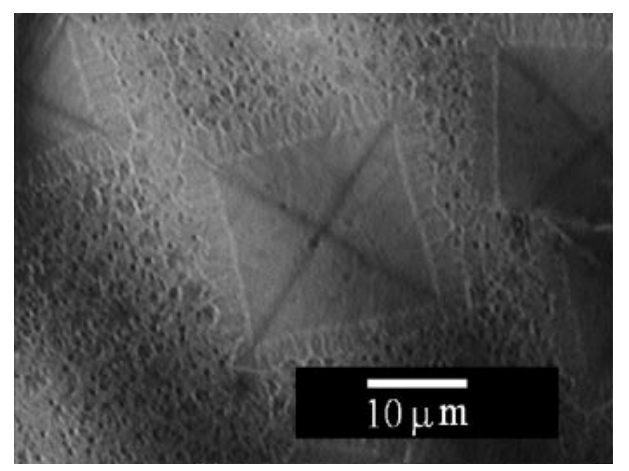

Figure 8. (a) Electron micrograph and (b) diffraction pattern of it-PB1 single crystal grown at $100^{\circ} \mathrm{C}$. The circle in (a) shows the selected area for the diffraction. (c) Optical micrograph of it-PB1 single crystals grown at $100{ }^{\circ} \mathrm{C}$ (reflection). tion pattern of a single crystal grown at $100^{\circ} \mathrm{C}$; optical micrograph of single crystals grown at $100^{\circ} \mathrm{C}$ is also shown. The net pattern with four-fold symmetry in Figure 8b shows the crystal in Figure 8a is a flat-on tetragonal single crystal. The trace of well facetted $\{100\}$ growth front is observed clearly in Figure 8a; the traces of facets appear even more clearly in the optical micrograph (Figure 8c). Also observed are the sector boundaries of $\{110\}$. The traces of growth fronts in Figures $8 \mathrm{a}$ and $8 \mathrm{c}$ indicate that the tetragonal single crystals are also well faceted at $100{ }^{\circ} \mathrm{C}$, and hence the tetragonal crystals grow by nucleation-con-

(a)

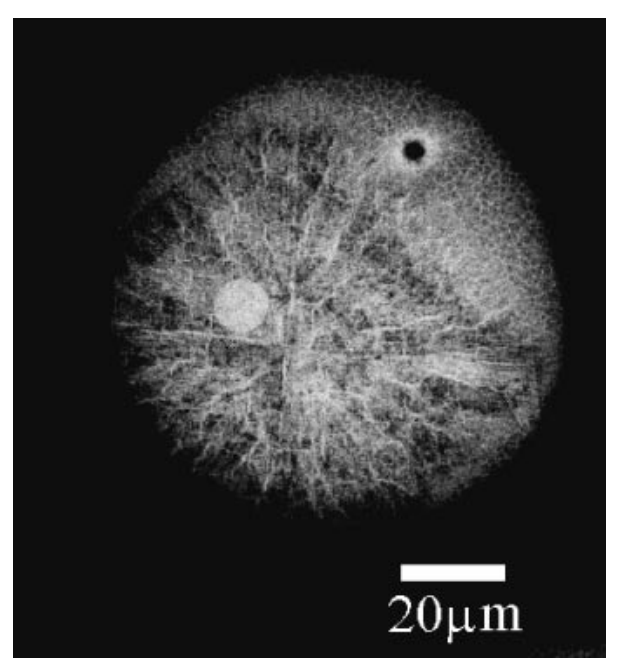

(b)

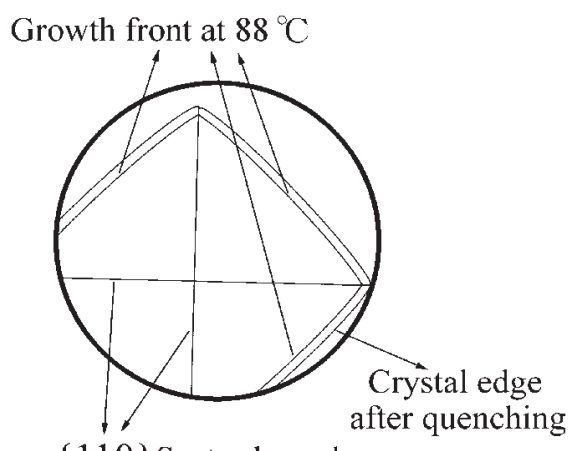

$\{110\}$ Sector boundary

(c)

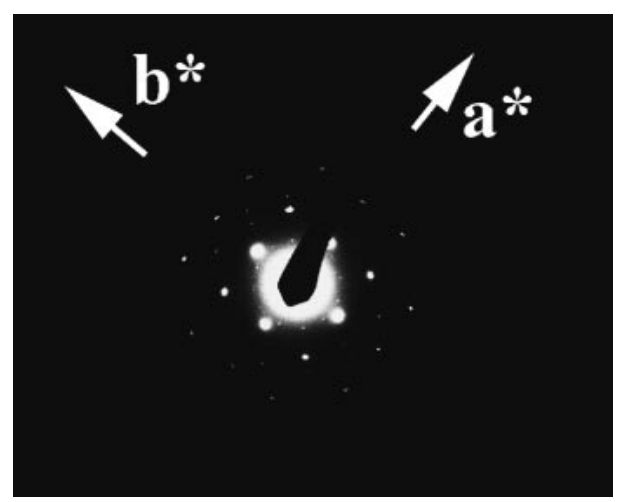

Figure 9. (a) Electron micrograph, (b) schematic illustration, and (c) diffraction pattern of $i t$-PB1 single crystal grown at $88^{\circ} \mathrm{C}$. 
trolled growth on $\{100\}$ plane at $100^{\circ} \mathrm{C}$.

A single crystal grown at $88^{\circ} \mathrm{C}$ also shows the same single-crystal net pattern as at $100{ }^{\circ} \mathrm{C}$ (Figure 9). The trace of the growth front of the single crystal, however, slightly rounds at the sides, while the corners still remain with four-fold symmetry. Sector boundaries in the $\{110\}$ directions are observed.

At a further lower temperature, $85^{\circ} \mathrm{C}$, the growth

(a)

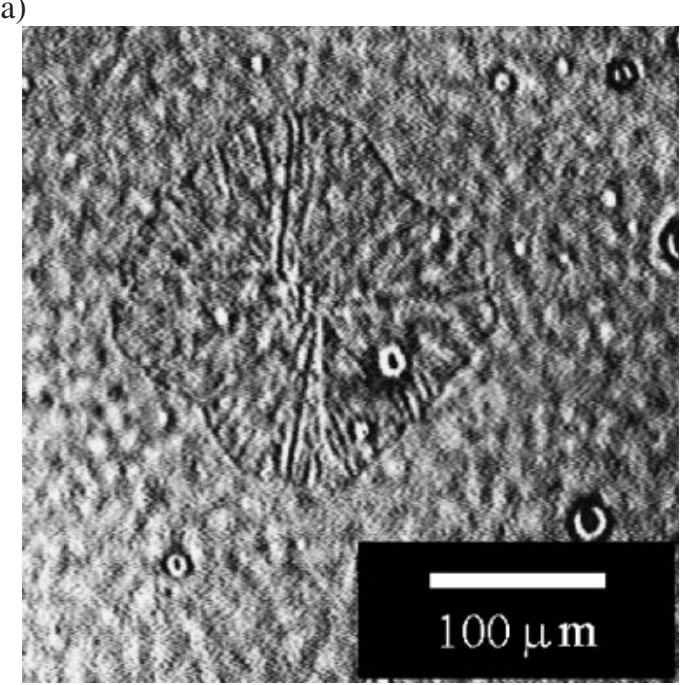

(b)

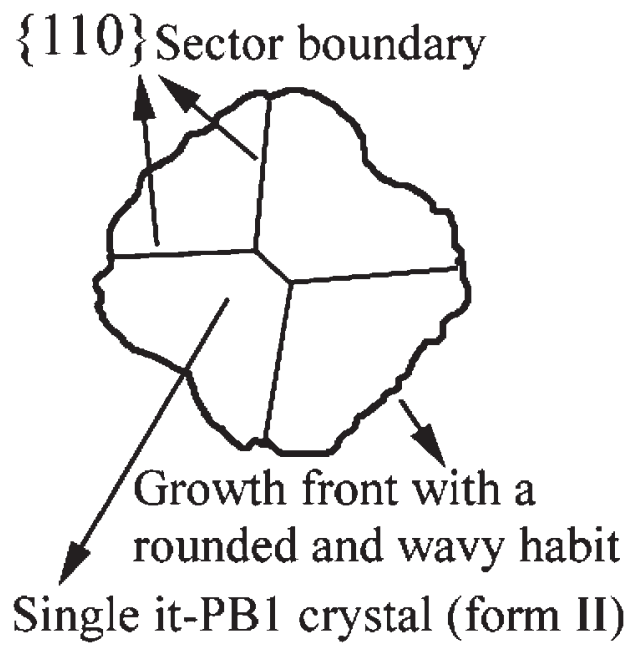

Figure 10. (a) In-situ optical micrograph (captured from video image; reflection) and (b) schematic illustration of an it-PB1 single crystal growing at $85^{\circ} \mathrm{C}$. front shows a rounded and wavy habit, indicating the growth face is kinetically roughened (Figure 10).

Therefore, it-PB1 tetragonal crystals have the kinetic roughening transition temperature around $85^{\circ} \mathrm{C}$; the growth mechanism changes from nucleation controlled to adhesive growth at crystallization temperatures lower than $85^{\circ} \mathrm{C}$ since the growth face becomes rough on the molecular scale.

\section{DISCUSSION}

\section{The Degree of Crystallinity}

So far, in the calculation of the values of side- and end-surface free energy $\sigma$ and $\sigma_{\mathrm{e}}$, we have assumed that crystal thicknesses $l_{\mathrm{c}}$ are equal to the long spacings $L$, i.e., the degree of crystallinity is 1.0. However, the long spacings $L$ is the sum of crystalline layer thickness $l_{\mathrm{c}}$ and inter-crystalline amorphous layer thickness $l_{\mathrm{a}}$.

Fu et al. showed that the crystallinity $\phi$ of it-PB1 takes an almost constant value of about 0.5 to 0.6 for a wide range of crystallization temperature ${ }^{20}$ and for different molecular weights, and also showed that the crystallinity $\phi$ remains almost the same value even after annealing. The relationship between $l_{\mathrm{c}}$ and $l_{\mathrm{a}}$ is given by the following equations:

$$
\begin{aligned}
& L=l_{\mathrm{c}}+l_{\mathrm{a}} \\
& \phi=\frac{l_{\mathrm{c}}}{L}
\end{aligned}
$$

Assuming that crystallinity $\phi$ is 0.5 , lamellar crystal thicknesses are half of the values listed in Table II; $\sigma$ and $\sigma_{\mathrm{e}}$ are obtained as $4.3 \times 10^{-3} \mathrm{~J} \mathrm{~m}^{-2}$ and $5.7 \times$ $10^{-2} \mathrm{~J} \mathrm{~m}^{-2}$, respectively. The value of $\sigma$ we obtained in this work is almost half the value of $\sigma=$ $7.2 \times 10^{-3} \mathrm{~J} \mathrm{~m}^{-2}$ estimated by Powers et al. ${ }^{21}$ from the value of $\Delta h_{\mathrm{f}}$ using an empirical relation ${ }^{22}$ which is given by the following equation.

$$
\frac{\sigma}{b_{0} \Delta h_{\mathrm{f}}}=0.106
$$

In Table III, the values of $\sigma, \sigma_{\mathrm{e}}, q, T_{\mathrm{m}}^{0}, K$ and $G_{0}$ obtained in this work are listed. The values of $\sigma, \sigma_{\mathrm{e}}$

\begin{tabular}{|c|c|c|c|c|c|c|c|c|c|c|c|}
\hline & $\begin{array}{c}\phi^{\mathrm{a}} \\
(-)\end{array}$ & $\begin{array}{l}T_{\mathrm{m}}{ }^{0} \\
\left({ }^{\circ} \mathrm{C}\right)\end{array}$ & $\begin{array}{c}K \\
\left(\mathrm{~K}^{2}\right) \\
\end{array}$ & $\begin{array}{c}G_{0} \\
\left(\mu \mathrm{m} \mathrm{s}^{-1}\right)\end{array}$ & $\begin{array}{c}a \\
(\mathrm{~nm})\end{array}$ & $\begin{array}{c}b \\
(\mathrm{~nm})\end{array}$ & $\begin{array}{c}a b \\
\left(\mathrm{~nm}^{2}\right)\end{array}$ & $\begin{array}{c}\sigma \sigma_{\mathrm{e}} \\
\left(\mathrm{J}^{2} \mathrm{~m}^{-4}\right)\end{array}$ & $\begin{array}{c}\sigma \\
\left(\mathrm{J} \mathrm{m}^{-2}\right)\end{array}$ & $\begin{array}{c}\sigma_{\mathrm{e}} \\
\left(\mathrm{J} \mathrm{m}^{-2}\right)\end{array}$ & $\begin{array}{c}q \\
(\mathrm{~J} / \text { stem })\end{array}$ \\
\hline $\begin{array}{c}i t-\mathrm{PB} 1 \\
\text { (this work) }\end{array}$ & $\begin{array}{l}1.0 \\
0.5\end{array}$ & 129.4 & $9.54 \times 10^{4}$ & $1.05 \times 10^{4}$ & 0.73 & 0.73 & $53.29 \times 10^{-2}$ & $245 \times 10^{-6}$ & $\begin{array}{r}2.15 \times 10^{-3} \\
4.3 \times 10^{-3}\end{array}$ & $\begin{array}{r}114 \times 10^{-3} \\
57 \times 10^{-3}\end{array}$ & $\begin{array}{r}1.21 \times 10^{-19} \\
6.1 \times 10^{-20}\end{array}$ \\
\hline$i t-\mathrm{PB} 1^{\mathrm{b}}$ & & 128.0 & $9.49 \times 10^{4 c}$ & $1.86 \times 10^{4 \mathrm{c}}$ & 0.745 & 0.745 & $55.50 \times 10^{-2}$ & & & & \\
\hline $\mathrm{PE}^{\mathrm{d}}$ & & 144.6 & $2.16 \times 10^{5}$ & $4.40 \times 10^{13}$ & 0.455 & 0.415 & $18.88 \times 10^{-2}$ & $1270 \times 10^{-6}$ & $14.1 \times 10^{-3}$ & $90.4 \times 10^{-3}$ & $3.4 \times 10^{-20}$ \\
\hline
\end{tabular}
and $q$ for PE are also listed for the sake of comparison.

Table III. Growth kinetics coefficients

${ }^{\mathrm{a}}$ Assumed crystallinity. $\quad{ }^{\mathrm{b}}$ From Ref $21{ }^{\mathrm{c}}$ Calculated from the data in Ref 17 , assuming $T_{\mathrm{m}}{ }^{0}$ is equal to $129.4{ }^{\circ} \mathrm{C}$. ${ }^{\mathrm{d}}$ From Ref 14 
The values of $\sigma$ and $\sigma_{\mathrm{e}}$ for it-PB1 are much smaller than those of $\sigma=14.1 \times 10^{-3} \mathrm{~J} \mathrm{~m}^{-2}$ and $\sigma_{\mathrm{e}}=97.8 \times$ $10^{-3} \mathrm{~J} \mathrm{~m}^{-2}$ for PE. However, the value of $q$ for $i t$-PB1 is much larger than that of $q=3.404 \times 10^{-20} \mathrm{~J} / \mathrm{stem}$ for PE. This is due to much larger area per unit stem, $a b$; the value of $a b=53.29 \times 10^{-2} \mathrm{~nm}^{2}$ for $i t$-PB1 is almost three times as large as the value of $a b=$ $18.88 \times 10^{-2} \mathrm{~nm}^{2}$ for PE. ${ }^{14}$ Both the side- and endsurface free energies of $i t$-PB1 can not reasonably be explained from the empirical relation.

\section{Kinetic Roughening Transition}

We have shown above that the crystallization temperature dependence of both growth rate and long spacings follow the equation derived from nucleation theory over the whole temperature range examined. Nucleation controlled growth requires flat growth faces, i.e., facets. However, TEM and OM showed that the morphology of it-PB1 crystals is rounded shape without any facets at crystallization temperatures lower than $85^{\circ} \mathrm{C}$, although lamellar single crystals possess facetted morphology in the temperature range of 88 to $110^{\circ} \mathrm{C}$.

The kinetic roughening transition can be described by the change in the kinetic length $d$. The length $d$ takes the values of the macroscopically large scale in the temperature range with facetted morphology; it gradually decreases with decreasing temperature, and reaches microscopically small values of the lattice constant scale at the kinetic roughening transition temperature, causing rough growth faces in the molecular scale. Hence a flat growth face required for nucleation and growth does not exist below the kinetic roughening transition temperature. However, the Eqs 1 and 5 derived from the nucleation theory still work for the rough surface growth (Figure 10). The same paradox pointed out in the growth of it-PS crystals in solution and in the melt is confirmed again in the growth of it-PB1 form II crystals from the melt. The inconsistency between the morphology and the temperature dependence of the growth rate is therefore not a specific problem to it-PS, but a general issue for the crystallization of polymers.

In order to expand the nucleation theory into rough surface growth, regime III growth is proposed ${ }^{1}$ as a growth mechanism for rough surface, where $d$ takes a value close to the unit stem width $(d-a)$. Regime III growth is reported in polyethylene (PE), ${ }^{23}$ polyoxymethylene (POM) ${ }^{24}$ and isotactic polypropylene (it$\mathrm{PP}) .^{25} \mathrm{In}$ regime III, layer addition is mainly governed by injection of stems at nucleation rate $i$, leading to growth rate $G_{\mathrm{III}}$. Step propagation becomes meaningless on rough surfaces, and propagation velocity $g$ no longer plays a role in growth process.

The growth rate $G_{\text {III }}$ is given by the following equa- tions:

$$
\begin{aligned}
G_{\mathrm{III}} & \cong b i a \\
& \propto \exp \left[-\frac{K_{\mathrm{III}}}{T \Delta T}\right] \\
K_{\mathrm{III}} & =\frac{4 b \sigma \sigma_{\mathrm{e}} T_{\mathrm{m}}^{0}}{k \Delta h_{\mathrm{f}}}=2 K_{\mathrm{II}}
\end{aligned}
$$

According to Hoffman, $d$ takes a value on the order of $2-2.5 a$ at the onset of regeime III. ${ }^{1}$ In this case, regime II-III transition takes place at the onset of regime III, around which the slope $K$ in $\ln G+$ $U / R\left(T-T_{\mathrm{V}}\right)$ vs. $1 /(T \Delta T)$ plot gradually changes and becomes the value twice as large as $K_{\mathrm{II}}$.

Regime II-III transition is caused by the change in $d$, and the change also causes the kinetic roughening transition. Therefore, the kinetic roughening transition should accompany regime II-III transition with a change of factor two in $K$. In either case of adhesive growth or regime III growth, kinetic roughening should accompany some transition in the temperature dependence of growth rate. This is, however, not the case in it-PS and it-PB1. The inconsistency between the morphology and the temperature dependence of growth rate still remains unresolved.

As a solution to the inconsistency in regime II-III transition, pinning barrier ${ }^{26-29}$ has been reevaluated in recent studies. ${ }^{17,30,31}$ The pinning barrier represents the trap by a stem shorter than $l^{*}=2 \sigma_{\mathrm{e}} / \Delta g$ ( $\Delta g$ is the free energy difference between crystal and liquid states per unit volume), the shortest stem length for crystal to grow. Further crystallization of a stable stem longer than $l^{*}$ attached to the shorter stem should proceed at the expense of a creation of new side surface. Crystallization is thereby interrupted by the short stem, and the pinning stem has to be removed to resume crystallization.

The growth rate limited by the pinning has an exponential dependence ${ }^{26-29}$ on supercooling of the form of $\exp \left[-K_{\text {pin }} / T \Delta T\right]$, which corresponds to the experimental dependence shown in Eq 2. The pinning barrier with the strong dependence on $\Delta T$ has been considered as an entropic barrier ${ }^{26-29}$ due to the increasing number of byroads, namely traps, with increasing stem length.

Pinning was originally proposed by Sadler and Gilmer $^{26-29}$ to explain the crystallization mechanism on thermally rough surface, where nucleation barrier was dismissed. In this case, pinning with the folding along the growth direction was assumed. Pinning was also introduced later by Doye and Frenkel to substrate completion process with folding along the growth face which is normal to the growth direction. ${ }^{30}$ This pinning does not require thermal roughening and 
hence can be compatible with nucleation theory.

By numerical calculation of a rate equation proposed by Sadler and Gilmer, ${ }^{27,29}$ Toda showed that the propagation velocity of steps, $g$, in the substrate completion process is given by the following equation: ${ }^{17}$

$$
g \propto \exp \left[-\left(0.2 \frac{\sigma c}{\sigma_{\mathrm{e}} a}+0.1\right) \frac{l^{*}}{c}\right]
$$

where $c$ is the size of the growth unit along the stem.

By comparing the pinning barrier, $\left(0.2 \sigma c / \sigma_{\mathrm{e}} a+\right.$ $0.1) l^{*} / c \equiv K_{\text {pin }} / T \Delta T$, with that of surface nucleation in Eq $2,4 b \sigma \sigma_{\mathrm{e}} T_{\mathrm{m}}^{0} / k \Delta h_{\mathrm{f}} T \Delta T \equiv K_{\text {nuc }} / T \Delta T$, the ratio $\alpha \equiv K_{\text {pin }} / K_{\text {nuc }}$ is given as follows:

$$
\alpha=0.1 \frac{k T}{\sigma_{\mathrm{e}} a b}+0.05 \frac{k T}{\sigma b c}
$$

Taking account pinning barrier $K_{\text {pin }} / T \Delta T$ into step propagation velocity $g$ in the substrate completion process, $G_{\mathrm{II}}$ is modified as follows:

$$
\begin{aligned}
G_{\mathrm{II}} & \cong b(2 i g)^{1 / 2} \\
& \propto \exp \left[-\frac{1}{T \Delta T} \frac{(1+\alpha)}{2} K_{\mathrm{nuc}}\right]
\end{aligned}
$$

The behavior of the kinetic length, $d$, is also modified. In the conventional nucleation theory, the $\Delta T$ dependence of $d$ is given by the following equation.

$$
\begin{aligned}
d & =\left(\frac{2 g}{i}\right)^{1 / 2} \\
& \propto \exp \left[\frac{K_{\mathrm{nuc}}}{2 T \Delta T}\right]
\end{aligned}
$$

In the pinning barrier treatment, however, the $\Delta T$ dependence of $d$ is modified as fallows.

$$
d \propto \exp \left[\frac{(1-\alpha) K_{\text {nuc }}}{2 T \Delta T}\right]
$$

The dependence expressed by Eq 18 shows a smaller change in $d$ with increasing $\Delta T$ than that expressed by Eq 17. This is due to the exponential dependence on $1 / T \Delta T$ of $g$ as well as $i$; the dependence of $g$ due to pinning barrier partially cancels that of $i$. (For the decrease of $d$ with increasing $\Delta T$, i.e., for the kinetic roughening transition to occur, $\alpha<1$ is required.).

Comparing modified $G_{\mathrm{II}}$ with $G_{\mathrm{III}} \propto \exp \left[-K_{\mathrm{III}} /\right.$ $T \Delta T]=\exp \left[-K_{\text {nuc }} / T \Delta T\right]$, it is evident that sufficiently large value of $\alpha$ makes Regime II-III transition difficult to be observed. In Regime II, the kinetic length $d$ is much larger by several orders of magnitude than $a(d \gg a)$ and $K=(1+\alpha) K_{\text {nuc }} / 2$. When $d$ gradually decreases with decreasing crystallization temperature and takes the value close to the unit stem width $(d \sim a)$, the growth is in Regime III and hence
$K=K_{\text {nuc }}$. At the onset of Regime III, i.e., at the regime II-III transition, the slope $K$ shows a change of factor of $2 /(1+\alpha)$. In the conventional nucleation theory, no pinning barrier is assumed; this corresponds to assuming $\alpha=0$, leading to the change of factor two in $K$. In the pinning barrier model, however, $\alpha$ is assumed to take a non-zero value, and the change is reduced from of factor 2 to of factor $2 /(1+\alpha)$. If $\alpha$ takes a large value sufficiently close to unity, the change in $K$ at the Regime II-III transition becomes too small to be experimentally observed.

Using the values of $\sigma$ and $\sigma_{\mathrm{e}}$ obtained above according to the nucleation theory, the ratio $\alpha$ is obtained as 0.13 for $i t$-PB1, with $a=b=7.3 \AA, c=$ $7.1 \AA, \sigma=4.3 \times 10^{-3} \mathrm{~J} \mathrm{~m}^{-2}, \sigma_{\mathrm{e}}=57 \times 10^{-3} \mathrm{~J} \mathrm{~m}^{-2}$, $T=100^{\circ} \mathrm{C}$. We assumed here the length $c$ to correspond to one turn of the helical chain. It-PB1 tetragonal phase has $11 / 3$ helix and the lattice constant $c_{0}$ consists of 11 monomers; one turn corresponds to $11 / 3$ monomers and $c$ is $c_{0} / 3=7.1 \AA$.

A more precise consideration yields a different value of $\sigma$ from that of $\sigma$ derived from the nucleation theory; the value of $\sigma$ depends on $\alpha$. According to Eq 16, the observed slope in $\ln G+U / R\left(T-T_{\mathrm{V}}\right)$ vs. $1 / T \Delta T$ plot, $K_{\mathrm{obs}}$, is given by the following equation:

$$
K_{\mathrm{obs}}=\frac{2(1+\alpha) b \sigma \sigma_{\mathrm{e}} T_{\mathrm{m}}^{0}}{k \Delta h_{\mathrm{f}}}
$$

Since the value $\sigma_{\mathrm{e}}$ is obtained from Gibbs-Thomson equation (Eq 8), independent of the nucleation theory, we can use the same value of $\sigma_{\mathrm{e}}$ for the pinning barrier model. The relationship between $\sigma$ obtained from the conventional nucleation theory, $\sigma_{\text {nuc }}$, and $\sigma$ derived using the concept of pinning barrier, $\sigma_{\text {pin }}$, is given as follows.

$$
(1+\alpha) \sigma_{\text {pin }}=\sigma_{\text {nuc }}
$$

The $\sigma$ in Eq 15 should be replaced by $\sigma_{\text {pin }}$, and Eq 15 is modified as follows.

$$
\alpha=0.1 \frac{k T}{\sigma_{\mathrm{e}} a b}+0.05 \frac{k T}{\sigma_{\mathrm{pin}} b c}
$$

$\alpha$ and $\sigma_{\text {pin }}$ have to satisfy both of Eqs 20 and $15^{\prime}$ and can be given by the following equations:

$$
\begin{gathered}
\alpha=\frac{\frac{0.1 k T}{a b \sigma_{\mathrm{e}}}+\frac{0.05 k T}{b c \sigma_{\mathrm{nuc}}}}{1-0.05 \frac{k T}{b c \sigma_{\mathrm{nuc}}}} \\
\sigma_{\text {pin }}=\frac{1}{1+\alpha} \sigma_{\text {nuc }}
\end{gathered}
$$

To determine the contribution $\alpha$, we need to estimate the size of the growth unit. The values of $a$ and $b$ are straightforwardly defined as the width and 


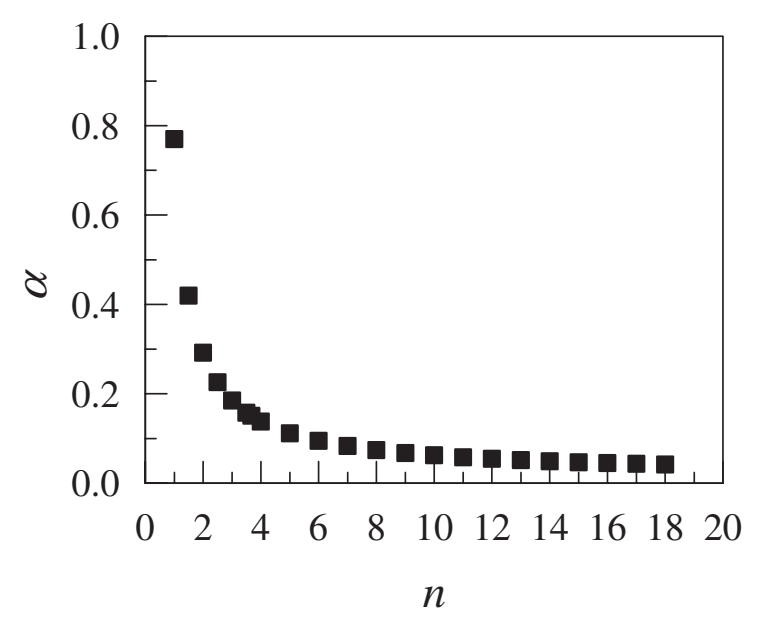

Figure 11. Plot of the ratio, $\alpha$, of pinning barrier to nucleation barrier as a function of the number, $n$, of monomers constituting a growth unit.

the thickness of a stem. However, there remains arbitrariness in estimating the value of $c$. The value $c$ corresponds to how many monomers constitute a growth unit. We need to consider the value $c$ in a range from a monomer size through the lattice constant (11 monomers) to persistence length ${ }^{13}$ (18 monomers). The choice of the size of growth unit means estimating the scale of cooperative motion when polymer chains are incorporated into crystal phase.

Figure 11 shows $\alpha$ as a function of $n$, the number of monomers constituting a growth unit. We obtain $\alpha=0.77,0.15,0.058$ and 0.04 for $n=1,11 / 3,11$ and 18, respectively, and all these values of $\alpha$ satisfy the condition $\alpha<1 . \alpha=0.77$ is too large a value for regime II-III transition to be observed, because the ratio of slope $K$ in Regime III $(d \sim a)$ to that in Regime II $(d \gg a), 2 /(1+\alpha)$, becomes as small as 1.14 for $\alpha=0.77$; this too close a value of $2 /(1+\alpha)$ to unity makes Regime II-III transition difficult to be experimentally observed. Therefore, if the crystallization of it-PB1 form II proceeds monomer by monomer, pinning barrier can explain invisible regime II-III transition. The size of growth unit is yet to be investigated, but pinning barrier can be a strong candidate for the solution to inconsistency between the morphology and the temperature dependence of growth rate.

Another solution to the inconsistency has been provided by applying the entropic barrier to the deposition of stems in the adhesive growth on rough surfaces. ${ }^{32}$ The entropic barrier is included in the preexponential factor as the entropy of melting which is assumed to be proportional to the lamellar thickness. This modified preexponential factor for adhesive growth is essentially the same with pinning barrier in the sense that the deposition of a stem next to a preexisting step requires a barrier proportional to the lamellar thickness.

\section{CONCLUSIONS}

Kinetic roughening transition was observed around $85^{\circ} \mathrm{C}$ in the crystallization of it-PB1 form II crystals from the melt; a flat growth face, facet, required for nucleation theory does not exist below $85^{\circ} \mathrm{C}$. On the other hand, the temperature dependence of growth rate and long spacings followed the nucleation theory in the temperature range of 52 to $111.9^{\circ} \mathrm{C}$. The dependences do not show any transition. While the important premise for nucleation theory is no longer satisfied below $85^{\circ} \mathrm{C}$, the nucleation theory in the growth of facetted crystals still seems to work for rough surfaces. This is the same paradox pointed out in the growth of it-PS crystals in solutions and from the melt. The inconsistency between the morphological change and temperature dependence of growth rate is no longer a problem specific to it-PS and hence should be addressed as a general problem for the crystallization of polymers. Still the size of the growth unit is to be investigated, the pinning barrier mechanism can be a candidate for the solution to the inconsistency.

Acknowledgment. The authors would like to acknowledge valuable discussions with Prof. Miyamoto (Kyoto University), Prof. Fukao (Kyoto Institute of Technology), and Dr. Tanzawa (Nagoya Institute of Technology).

\section{REFERENCES}

1. J. D. Hoffman and R. L. Miller, Polymer, 38, 3151 (1997).

2. J. J. Point and J. J. Janimak, Polymer, 39, 7123 (1998).

3. J. J. Point, M. C. Colet, and M. Dosiere, J. Polym. Sci., Polym. Phys. Ed., 24, 357 (1986).

4. Y. Saito, "Statistical Physics of Crystal Growth," World Scientific, Singapore, 1996.

5. Y. Tanzawa, Polymer, 33, 2659 (1992).

6. Y. Miyamoto, Y. Tanzawa, H. Miyaji, and H. Kiho, Polymer, 33, 2496 (1992).

7. G. Natta, P. Pino, P. Corradini, F. Danusso, E. Mantica, G. Mazzanti, and G. Moraglio, J. Am. Chem. Soc., 77, 1708 (1955).

8. G. Natta, Makromol. Chem., 35, 94 (1960).

9. A. Turner Jones, Polym. Lett., 1, 455 (1963).

10. V. Petraccone, B. Pirozzi, A. Frasci, and P. Corradini, Eur. Polym. J., 12, 323 (1976).

11. G. Natta, P. Corradini, and I. W. Bassi, Nuovo Cimento, Suppl., 15, 52 (1960).

12. K. Tashiro, A. Saiani, S. Miyashita, Y. Chatani, and H. Tadokoro, Polym. Prepr. Jpn., 47, 3869 (1998).

13. J. Brandrup and E. H. Immergut, Ed., "Polymer Handbook," Wiley-Interscience, New York, N.Y., 1966.

14. J. D. Hoffman, G. T. Davis, and J. I. Lauritzen Jr., "Treatise on Solid State Chemistry,” Hannay, N.B., 1976, p 497. 
15. H. W. Starkweather, Jr. and G. A. Jones, J. Polym. Sci., Part B: Polym. Phys., 24, 1509 (1986).

16. U. Leute and W. Dollhopf, Colloid Polym. Sci., 261, 299 (1983).

17. A. Toda, J. Chem. Phys., 118, 8446 (2003).

18. R. D. Icenogle, J. Polym. Sci., Polym. Phys. Ed., 23, 1369 (1985).

19. T. Kamijo, M. Tosaka, M. Tsuji, and S. Kohjiya, Polym. Prepr. Jpn., 48, 912 (1999).

20. Q. Fu, B. Heck, G. Strobl, and Y. Thomann, Macromolecules, 34, 2502 (2001).

21. J. Powers, J. D. Hoffman, J. J. Weeks, and F. A. Quinn Jr., J. Res. Natl. Bur. Stand. (U.S.), 69A, 335 (1965).

22. J. D. Hoffman and J. J. Weeks, J. Chem. Phys., 37, 1723 (1962).

23. J. P. Armisted and J. D. Hoffman, Macromolecules, 35, 3895 (2002).
24. J. D. Hoffman, Polymer, 24, 3 (1983).

25. E. J. Clark and J. D. Hoffman, Macromolecules, 17, 878 (1984).

26. D. M. Sadler and G. H. Gilmer, Polymer, 25, 1446 (1984).

27. D. M. Sadler and G. H. Gilmer, Phys. Rev. Lett., 56, 2708 (1986).

28. D. M. Sadler, Nature, 326, 174 (1987).

29. D. M. Sadler and G. H. Gilmer, Phys. Rev. B, 38, 5684 (1984).

30. J. P. K. Doye and D. Frenkel, J. Chem. Phys., 110, 2692 (1999).

31. J. P. K. Doye and D. Frenkel, J. Chem. Phys., 110, 7073 (1999).

32. H. Miyaji, Y. Miyamoto, K. Taguchi, A. Hoshino, M. Yamashita, O. Sawanobori, and A. Toda, J. Macromol. Sci. B, 42, 867 (2003). 